\title{
Reliability-Based Analysis and Design of Strip Footings against Bearing Capacity Failure
}

\author{
Dalia S. Youssef Abdel Massih; Abdul-Hamid Soubra; and Bak Kong Low
}

\begin{abstract}
This paper presents a reliability-based approach for the analysis and design of a shallow strip footing subjected to a vertical load with or without pseudostatic seismic loading. Only the punching failure mode of the ultimate limit state is studied. The deterministic models are based on the upper-bound method of the limit analysis theory. The random variables used are the soil shear strength parameters and the horizontal seismic coefficient. The Hasofer-Lind reliability index and the failure probability are determined. A sensitivity analysis is also performed. The influence of the applied footing load on the reliability index and the corresponding design point is presented and discussed. It was shown that the negative correlation between the soil shear strength parameters highly increases the reliability of the foundation and that the failure probability is highly influenced by the coefficient of variation of the angle of internal friction of the soil and the horizontal seismic coefficient. For design, an iterative procedure is performed to determine the breadth of the footing for a target failure probability.
\end{abstract}

CE Database subject headings: Bearing capacity; Earthquake loads; Limit analysis; Probability; Reliability; Shallow foundations; Punching; Failure loads.

\section{Introduction}

In the analysis and design of geotechnical structures, all the input data have some degree of uncertainty and, thus, may be considered as random variables or stochastic processes. Geotechnical engineers have always recognized the presence of uncertainty in their analysis and design. However, traditional deterministic models simplify the problem by considering the uncertain parameters to be deterministic and by accounting for the uncertainties through the use of a global safety factor, which is essentially a "factor of ignorance." This factor is derived based on past experience and does not reflect the inherent uncertainty of each parameter. A reliability-based analysis or design is more rational, since it takes into account the inherent uncertainty of each input variable. Nowadays, this is possible because of the improvement of our knowledge on the statistical properties of the soil (Phoon and Kulhawy 1999).

In the last decade, the reliability-based analysis has been extensively applied to the slope stability problem [see, for instance, Christian et al. (1994); Low and Tang (1997b,c); Low et al. (1998); Hassan and Wolff (1999); El-Ramly et al. (2002), (2003); among others]. However, very few authors have investigated the reliability-based analysis and design of foundations. Some (Griffiths and Fenton 2001; Fenton and Griffiths 2002, 2003, 2005; Griffiths et al. 2002; Przewlocki 2005; Popescu et al. 2005) modeled the uncertain parameters as random processes and examined the effect of the spatial variability of these parameters on the settlement or on the bearing capacity of foundations. Most of these studies considered the uncertainty of one single parameter (such as the soil elastic modulus, the cohesion, or the angle of internal friction of the soil). Others modeled the uncertainties of the different parameters as random variables and conducted reliability-based analysis using formulas of the bearing capacity factors (Cherubini 2000; Low and Phoon 2002). These approaches have the merit of simplicity, but also some shortcomings because they are based on approximate theoretical formulations.

In this study, a reliability-based analysis and design of a strip foundation resting on a $c-\varphi$ soil is presented. Two performance functions may characterize the footing behavior: The serviceability limit state and the ultimate limit state. Only the punching failure mode of the ultimate limit state is analyzed herein. Two loading cases are considered. The first examines the case of a strip footing subjected to a vertical load only; the second considers the case of a vertically loaded strip footing situated in a seismic area and subjected to a pseudostatic seismic loading. Two rigorous deterministic limit analysis models are used. The uncertainties of the soil shear strength parameters and the seismic coefficient are modeled as random variables. The basic concepts of the theory of reliability are described next, followed by the two deterministic models, and discussions of the probabilistic numerical results based on these models. 


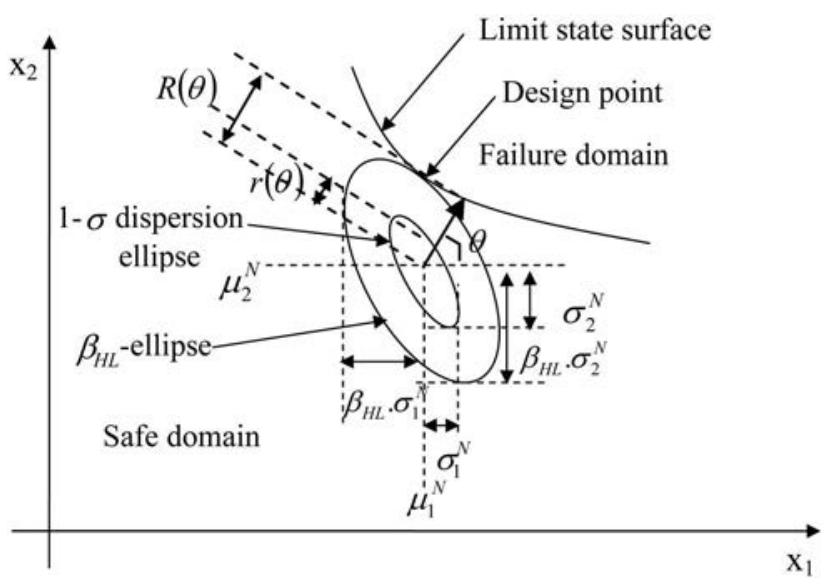

Fig. 1. Design point and equivalent normal dispersion ellipses in the space of two random variables

\section{Basic Reliability Concepts}

Two different measures are commonly used in the literature to describe the reliability of a structure: The reliability index and the failure probability.

\section{Reliability Index}

The reliability index of a geotechnical structure is a measure of the safety that takes into account the inherent uncertainties of the input variables. A widely used reliability index is the Hasofer and Lind (1974) index defined as the shortest distance from the mean value point of the random variables to the limit state surface in units of directional standard deviations, namely $\beta_{\mathrm{HL}}=\min [R(\theta) / r(\theta)]$ (Fig. 1). Its matrix formulation is (Ditlevsen 1981)

$$
\beta_{\mathrm{HL}}=\min _{x \in F} \sqrt{(x-\mu)^{T} C^{-1}(x-\mu)}
$$

in which $x=$ vector representing the $n$ random variables; $\mu=$ vector of their mean values; $C=$ covariance matrix; and $F=$ failure region. The minimization of Eq. (1) is performed subject to the constraint $G(x) \leqslant 0$ where the limit state surface $G(x)=0$, separates the $n$ dimensional domain of random variables into two regions: A failure region $F$ represented by $G(x) \leqslant 0$ and a safe region given by $G(x)>0$.

The classical approach for computing $\beta_{\mathrm{HL}}$ by Eq. (1) is based on the transformation of the limit state surface into the rotated space of standard normal uncorrelated variates. The shortest distance from the transformed failure surface to the origin of the reduced variates is the reliability index $\beta_{\mathrm{HL}}$.

An intuitive interpretation of the reliability index was suggested in Low and Tang (1997a, 2004), where the concept of an expanding ellipse (Fig. 1) led to a simple method of computing the Hasofer-Lind reliability index in the original space of the random variables using an optimization tool available in most spreadsheet software packages. When there are only two uncorrelated nonnormal random variables $x_{1}$ and $x_{2}$, these variables span a two-dimensional random space, with an equivalent onesigma dispersion ellipse [corresponding to $\beta_{\mathrm{HL}}=1$ in Eq. (1) without the min.], centered at the mean values $\left(\mu_{1}^{N}, \mu_{2}^{N}\right)$ and whose axes are parallel to the coordinate axes of the original space. For correlated variables, a tilted ellipse is obtained. Low and Tang (1997a, 2004) reported that the Hasofer-Lind reliability index $\beta_{\mathrm{HL}}$ may be regarded as the codirectional axis ratio of the smallest ellipse (which is either an expansion or a contraction of the $1-\sigma$ ellipse) that just touches the limit state surface to the $1-\sigma$ dispersion ellipse. They also stated that finding the smallest ellipsoid that is tangent to the limit state surface is equivalent to finding the most probable failure point.

\section{Failure Probability}

Given a vector of $n$ random variables $X$ and a performance function defined by $G(x)$, the failure probability $P_{f}$ is defined by

$$
P_{f}=\int_{G(x) \leqslant 0} f(x) d x
$$

where $f(x)=$ joint probability density function of the random variables $X$. By introducing the indicator function $I(x)$ defined as

$$
I(x)= \begin{cases}1 & \text { if } G(x) \leqslant 0 \\ 0 & \text { elsewhere }\end{cases}
$$

The integral that gives the failure probability [Eq. (2)] can be written as

$$
P_{f}=\int_{\Omega} I(x) f(x) d x
$$

where $\Omega=$ total domain. A brief description of the methods used in this paper for the evaluation of this integral (i.e., FORM, MC, and IS) follows.

\section{First-Order Reliability Method}

From the first-order reliability method (FORM) and the HasoferLind reliability index $\beta_{\mathrm{HL}}$, one can approximate the failure probability as follows:

$$
P_{f} \approx \Phi\left(-\beta_{\mathrm{HL}}\right)
$$

where $\Phi(\cdot)=$ cumulative distribution function of a standard normal variable. In this method, the limit state function is approximated by a hyperplane tangent to the limit state surface at the design point.

\section{Crude Monte Carlo Simulation}

Monte Carlo is the most robust simulation method in which samples are generated with respect to the probability density of each variable. For each sample, the response of the system is calculated. An unbiased estimator of the failure probability is given by

$$
\widetilde{P}_{f}=\frac{1}{N} \sum_{i=1}^{N} I\left(x_{i}\right)
$$

where $N=$ number of samples and $I(x)$ is as defined in Eq. (3). The coefficient of variation of the estimator is given by

$$
\operatorname{CoV}\left(\widetilde{P}_{f}\right)=\sqrt{\frac{\left(1-P_{f}\right)}{P_{f} N}}
$$

\section{Importance Sampling Simulation}

Generally, for a given target of the coefficient of variation, the crude Monte Carlo simulation requires a large number of samples, i.e., a large computation time. This is especially the case for small values of the failure probability $P_{f}$. The importance sampling (IS) simulation method is a more efficient approach; it requires fewer 
sample points than the crude Monte Carlo method. In this approach, the initial sampling density is shifted to the design point in order to concentrate the samples in the region of greatest probability density within the zone defined by $G(x) \leqslant 0$. The design point may be determined by using any of the classical methods such as Rackwitz-Fiessler algorithm (Rackwitz-Fiessler 1978), Low and Tang's ellipsoid approach (Low and Tang 1997a, 2004), etc. An estimator of the failure probability $P_{f}$ is obtained as follows (Melchers 1999):

$$
\widetilde{P}_{f}=\frac{1}{N} \sum_{i=1}^{N} I\left(v_{i}\right) \frac{f\left(v_{i}\right)}{h\left(v_{i}\right)}
$$

where $h(\cdot)=$ new sampling density centred at the design point, and $v=$ vector of sample values with probability density function $h(\cdot)$. The coefficient of variation of the estimator is given by (Melchers 1999)

$$
\operatorname{COV}\left(\widetilde{P}_{f}\right)=\frac{1}{P_{f}} \sqrt{\frac{1}{N}\left[\frac{1}{N} \sum_{i=1}^{N}\left(I\left(v_{i}\right) \frac{f\left(v_{i}\right)}{h\left(v_{i}\right)}\right)^{2}-\left(P_{f}\right)^{2}\right]}
$$

\section{Sensitivity Factors}

The sensitivity factors convey the relative importance of the random variables in affecting reliability. The "omission sensitivity factor" is used in this paper. It gives the relative error in the reliability index when a random variable is replaced by its deterministic mean value. It is given by

$$
\gamma_{i}\left(\mu_{i}\right)=\frac{\beta_{\mathrm{HL}}\left(x_{i}=\mu_{i}\right)}{\beta_{\mathrm{HL}}}
$$

where $\beta_{\mathrm{HL}}=$ value of the reliability index calculated when all parameters are considered as random variables, and $\beta_{\mathrm{HL}}\left(x_{i}=\mu_{i}\right)$ $=$ reliability index determined when $x_{i}$ is replaced by its deterministic mean value.

\section{Reliability Analysis of Shallow Strip Foundations}

The aim of this paper is to perform a reliability analysis of a strip footing resting on a $c-\varphi$ soil and subjected to a vertical load. The case of a vertically loaded strip footing situated in a seismic area is also studied. Quasi-static representation of earthquake effects using the seismic coefficient concept is adopted. The earthquake acceleration for both the soil and the structure is assumed to be the same: Only the horizontal seismic coefficient $K_{h}$ is considered, the vertical seismic coefficient often being disregarded. The deterministic models are based on the upper-bound method of the limit analysis theory (Soubra 1999). Due to uncertainties in soil shear strength parameters and horizontal seismic coefficient, the cohesion $c$, the angle of internal friction $\varphi$, and the seismic coefficient $K_{h}$ are considered as random variables. The performance function used in the reliability analysis is defined with respect to the bearing failure of the soil. It is given as follows:

$$
G=\frac{P_{u}}{P_{S}}-1
$$

where $P_{u}=$ ultimate foundation load, and $P_{S}=$ applied load. One may use another performance function as $G=P_{u}-P_{S}$. However, this leads exactly to the same value of the reliability index since the Hasofer-Lind reliability index does not vary with alternative but equivalent definitions of the performance function. Notice,

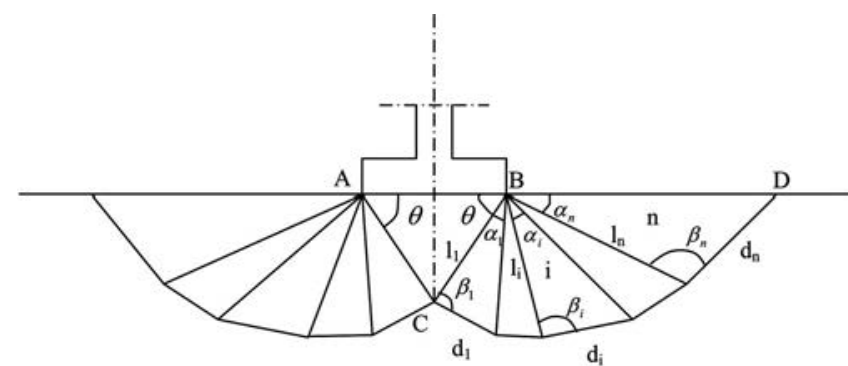

Fig. 2. Failure mechanism $M 1$ for static bearing capacity analysis

however, that the first definition has an advantage over the second one since it makes it possible to introduce the concept of the safety factor as follows: $G=F-1$.

\section{Limit Analysis Models}

Two deterministic models are used in this paper. In these models, the upper-bound theorem of limit analysis is applied to the bearing capacity problem of a strip footing using two kinematically admissible failure mechanisms referred to in the following as $M 1$ and M2. These mechanisms were presented by Soubra (1999). The approach is simple and self-consistent and it obtains rigorous upper-bound solutions in the framework of limit analysis theory. Although the results given by this approach are upper-bound solutions, they are the smallest upper-bounds against the available ones given by rigid block mechanisms. In some cases (i.e., for weightless soil), they are the exact solutions, since they are equal to the results given by the lower-bound method.

$M 1$ is a translational symmetrical multiblock failure mechanism (Fig. 2) and is used for the computation of the bearing capacity of a vertically loaded footing. M2 is a translational nonsymmetrical multiblock failure mechanism and is suitable for the calculation of the bearing capacity of vertically loaded foundations situated in seismic areas by a pseudostatic approach (Fig. 3). The calculation of the bearing capacity is performed by equating the total rate at which work is done by the foundation load, the soil weight in motion, the horizontal seismic loads (in the case of seismic loading), and the ground surface surcharge to the total rate of energy dissipation along the lines of velocity discontinuities. For both $M 1$ and $M 2$, it is found that an upper-bound on the bearing capacity of the soil is given as follows:

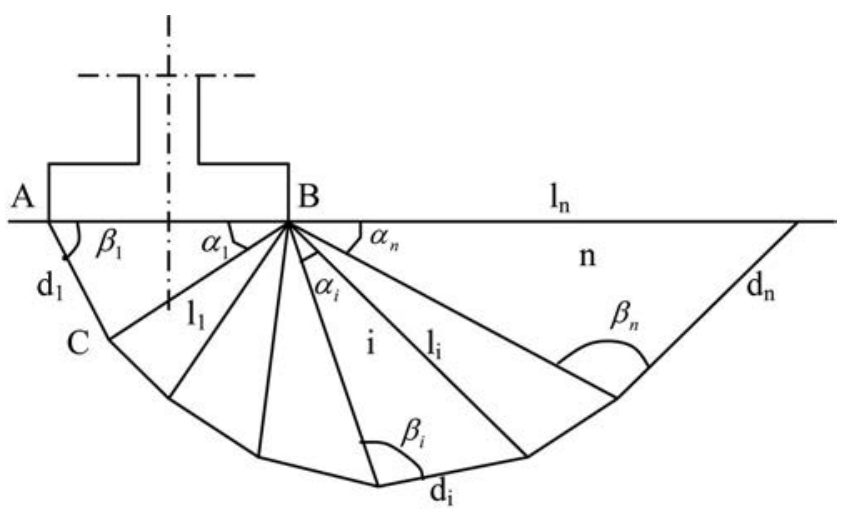

Fig. 3. Failure mechanism $M 2$ for seismic bearing capacity analysis 


$$
P_{u}=\frac{1}{2} \gamma B^{2} N_{\gamma}+q B N_{q}+c B N_{c}
$$

in which the bearing capacity factors $N_{\gamma}, N_{q}$, and $N_{c}$ can be expressed in terms of the geometrical parameters of each mechanism. For the $M 1$ mechanism, these factors are given as follows:

$$
\begin{gathered}
N_{\gamma}=-\left(f_{1}+f_{2}\right) \\
N_{q}=-f_{3} \\
N_{c}=2\left(f_{4}+f_{5}+f_{6}\right)
\end{gathered}
$$

where the expressions of $f_{i}(i=1, \ldots, 6)$ are given in Appendix I. For the $M 2$ mechanism, the bearing capacity factors are given by

$$
\begin{gathered}
N_{\gamma}=-\frac{1}{\sin \left(\beta_{1}-\varphi\right)+K_{h} \cos \left(\beta_{1}-\varphi\right)}\left(g_{1}+K_{h} g_{2}\right) \\
N_{q}=-\frac{1}{\sin \left(\beta_{1}-\varphi\right)+K_{h} \cos \left(\beta_{1}-\varphi\right)}\left(g_{3}+K_{h} g_{4}\right) \\
N_{c}=\frac{1}{\sin \left(\beta_{1}-\varphi\right)+K_{h} \cos \left(\beta_{1}-\varphi\right)}\left(g_{5}+g_{6}\right)
\end{gathered}
$$

where the expressions of $g_{i}(i=1, \ldots, 6)$ are given in Appendix II. The ultimate load of the foundation is obtained by minimization of Eq. (12) with regard to the mechanism's geometrical parameters. For further details on the failure mechanisms, the reader can refer to Soubra (1999).

\section{Ellipsoid Approach via Spreadsheet}

Low and Tang (1997a, 2004) showed that the minimization of the Hasofer-Lind reliability index can be efficiently carried out in the Excel spreadsheet environment. The spreadsheet approach is simple and easy to understand because it works in the original space of random variables and does not require the additional step of transforming $x$ to $u$ where $u$ is a transformed vector of the random variables in the uncorrelated Gaussian space. However, the optimization in original space is not preferred from a computational perspective (STRUREL 1991). This is because optimization in standardized space is mathematically more desirable in nonlinear optimization. For example, when minimizing the quadratic form of Eq. (1) in the original space, in some cases the correct solution is obtained only when the solver option "use automatic scaling" is activated. As an alternative, Cholesky factorization of the convariance matrix can be used. The robustness of the latter approach is investigated in Phoon (2004).

When the random variables are nonnormal, the RackwitzFiessler equivalent normal transformation was used to compute the equivalent normal mean $\mu_{x}^{N}$ and the equivalent normal standard deviation $\sigma_{x}^{N}$. The iterative computations of $\mu_{x}^{N}$ and $\sigma_{x}^{N}$ for each trial design point are automatic during the constrained optimization search.

In the present paper, by the Low and Tang method, one literally sets up a tilted ellipsoid in the Excel spreadsheet and minimizes the dispersion ellipsoid subject to the constraint that it be tangent to the limit state surface using the Excel Solver with the automatic scaling option. Eq. (1) may be rewritten as (Low and Tang 1997c, 2004)

$$
\beta_{\mathrm{HL}}=\min _{x \in F} \sqrt{\left[\frac{x-\mu_{x}^{N}}{\sigma_{x}^{N}}\right]^{T}[R]^{-1}\left[\frac{x-\mu_{x}^{N}}{\sigma_{x}^{N}}\right]}
$$

in which $[R]^{-1}=$ inverse of the correlation matrix. This equation will be used [instead of Eq. (1)] since the correlation matrix $[R]$ displays the correlation structure more explicitly than the covariance matrix $[C]$.

Additional information on Solver's options and algorithms can be found in the Microsoft Excel Solver's help file and at www.solver.com. The FORM implementation procedure in the spreadsheet is described in Low (2005) and Low and Tang (1997a,b,c, 2004). Some Excel files are available at http:// alum.mit.edu/www/bklow.

\section{Probabilistic Numerical Results}

For both $M 1$ and $M 2$ failure mechanisms, the numerical results presented in this paper consider the case of a shallow strip foundation with breadth $B=2 \mathrm{~m}$. The soil has a unit weight of $18 \mathrm{kN} / \mathrm{m}^{3}$. No surcharge loading $(q=0)$ is considered in the analysis. Different values of the coefficients of variation of the angle of internal friction and cohesion are presented in the literature. For most soils, the mean value of the effective angle of internal friction is typically between 20 and $40^{\circ}$. Within this range, the corresponding coefficient of variation as proposed by Phoon and Kulhawy (1999) is essentially between 5 and 15\%. For the effective cohesion, the coefficient of variation varies between 10 and $70 \%$ (Cherubini 2000). For the coefficient of correlation, Harr (1987) has shown that a correlation exists between the effective cohesion $c$ and the effective angle of internal friction $\varphi$. The results of Wolff (1985) $\left(\rho_{c, \varphi}=-0.47\right)$, Yuceman et al. (1973) $\left(-0.49 \leqslant \rho_{c, \varphi} \leqslant-0.24\right)$, Lumb (1970) $\left(-0.7 \leqslant \rho_{c, \varphi} \leqslant-0.37\right)$, and Cherubini (2000) $\left(\rho_{c, \varphi}=-0.61\right)$ are among the ones cited in the literature. In this paper, the illustrative values used for the statistical moments of the shear strength parameters and their coefficient of correlation $\rho_{c, \varphi}$ are as follows: $\mu_{c}=20 \mathrm{kPa}, \mu_{\varphi}=30^{\circ}$, $\mathrm{COV}_{c}=20 \%, \mathrm{COV}_{\varphi}=10 \%$, and $\rho_{c, \varphi}=-0.5$. These values are within the range of the values cited above.

\section{Mechanism M1}

For the configuration presented above, the ultimate footing load determined for the mean values of the soil shear strength parameters is $P_{u}=2,136.72 \mathrm{kN} / \mathrm{m}$. For the probability distribution of the random variables, two cases are studied. In the first case, referred to as normal variables, $c$ and $\varphi$ are considered as normal variables. In the second case, referred to as nonnormal variables, $c$ is assumed to be lognormally distributed while $\varphi$ is assumed to be bounded and a beta distribution is used (Fenton and Griffiths 2003). The parameters of the beta distribution are determined from the mean value and standard deviation of $\varphi$ (Haldar and Mahadevan 2000). For both cases, correlated or uncorrelated variables are considered.

\section{Probabilistic Failure Surface}

The conventional deterministic approach for the calculation of a safety factor or an ultimate load on a soil mass is based on the minimization of these functions over a range of trial failure surfaces. The surface of minimum factor of safety or ultimate load is referred to as the critical deterministic surface. A common approach to determine the reliability of a stressed soil mass is based 
on the calculation of the reliability index $\beta_{\mathrm{HL}}$ corresponding to this surface (Christian et al. 1994). In this paper, a more rigorous approach is used. It consists of the determination of the reliability index by minimizing the quadratic form of Eq. (19) not only with respect to the random variables, but also with respect to the geometrical parameters of the failure mechanism $\left(\theta, \alpha_{i}, \beta_{i}\right)$. Twelve rigid blocks (i.e., $n=12$ in Fig. 2 ) are considered for each side of the footing. Therefore, the minimization is performed with respect to 27 parameters $\left(\theta, \alpha_{i}, \beta_{i}, c, \varphi\right)$. The surface obtained corresponding to the minimum reliability index is referred to here as the critical probabilistic surface. The reliability index calculated with respect to the critical probabilistic surface is smaller (i.e., more critical) than the one calculated by using the critical deterministic surface. Had one considered the deterministic critical surface, a slightly higher reliability index value of 3.49 would have been obtained instead of 3.27 for the case with normal uncorrelated soil shear strength parameters. In all subsequent results, for both the $M 1$ and $M 2$ mechanisms, the reliability index is calculated using the probabilistic failure surface.

\section{Reliability Index and Sensitivity Factors}

Table 1 presents the Hasofer-Lind reliability index and the corresponding design point for different values of the vertical applied load $P_{S}$ varying from small values up to the deterministic ultimate load. This ultimate load is the one for which the design point is equal to the mean point for normal variables and equivalent normal mean point for nonnormal variables. The table also gives the omission sensitivity factors of the angle of internal friction and the cohesion. All these results are presented for normal and nonnormal, correlated and uncorrelated shear strength parameters. For all cases, the reliability index decreases with the increase of the applied load $P_{S}$ (i.e., with the decrease of the safety factor $\left.F=P_{u} / P_{S}\right)$ until it vanishes for an applied load equal to the deterministic ultimate load. This case corresponds to a deterministic state of failure for which $F=1$ using the mean values of the random variables and the failure probability is equal to $50 \%$.

The comparison of the results of correlated variables with those of uncorrelated variables (Fig. 4) shows that the reliability indices corresponding to uncorrelated variables are smaller than those of negatively correlated variables for both normal and nonnormal variables. One can conclude that assuming uncorrelated shear strength parameters is conservative in comparison to assuming negatively correlated shear strength parameters. For instance, when the safety factor is equal to 4.27 (i.e., $P_{S}=500 \mathrm{kN} / \mathrm{m}$ ), the reliability index increases by $40 \%$ if the variables $c$ and $\varphi$ are considered to be negatively correlated. Fig. 4 also shows that for small values of the safety factor, the results of normal and nonnormal variables are nearly identical. A difference appears for large values of the safety factor.

From Table 1, when $P_{S}=700 \mathrm{kN} / \mathrm{m}$, the most probable failure point for uncorrelated and correlated normal variables is found to be at $\left(c^{*}=12.45 \mathrm{kPa}, \varphi^{*}=22^{\circ}\right)$ and $\left(c^{*}=16.55 \mathrm{kPa}, \varphi^{*}=19.88^{\circ}\right)$, respectively. These are the points of tangency of the $\beta_{\mathrm{HL}}-\sigma$ ellipses with the limit state surface. Notice that the limit state surface divides the combinations of $(c, \varphi)$ that would lead to failure from the combinations that would not. The $(c, \varphi)$ values defining the limit state surface are obtained by searching $c$ (or $\varphi$ ) for a prescribed $\varphi$ (or $c$ ) that achieve both the conditions (i) a minimum ultimate load $P_{u}$ and (ii) a safety factor $F=P_{u} / P_{S}=1$. For this purpose, a numerical procedure was coded in Microsoft Excel Visual Basic. It calls the Excel Solver iteratively in order to simultaneously satisfy the two conditions above. Fig. 5 provides graphical representation of the reliability analysis for both corre- lated and uncorrelated shear strength parameters in the physical space of the random variables. One can easily see that negative correlation between shear strength parameters rotates the major axis of the ellipse from the horizontal direction.

The values $\left(c^{*}\right.$ and $\left.\varphi^{*}\right)$ of the design points corresponding to different values of the vertical applied load can give an idea about the partial safety factors of each of the strength parameters $c$ and $\tan \varphi$ as follows:

$$
\begin{gathered}
F_{c}=\frac{\mu_{c}}{c^{*}} \\
F_{\varphi}=\frac{\tan \left(\mu_{\varphi}\right)}{\tan \varphi^{*}}
\end{gathered}
$$

For uncorrelated shear strength parameters, the values of $c^{*}$ and $\varphi^{*}$ at the design point are smaller than their respective mean values and increase with the increase of the applied load. For negatively correlated shear strength parameters, $c^{*}$ slightly exceeds the mean for some values of the applied load.

The values of the omission sensitivity factors have shown that the effect of the randomness of the angle of internal friction on the reliability index is much more pronounced than that of the cohesion, especially for the case of nonnormal random variables.

\section{Failure Probability}

Figs. 6 and 7 present, respectively, the failure probability and the corresponding coefficient of variation as a function of the number of samples for both MC and IS simulations when the soil shear strength parameters are normal and uncorrelated and the footing applied load is equal to $700 \mathrm{kN} / \mathrm{m}$. In this paper, MC and IS simulations were performed in the standardized space of uncorrelated variables. Hence, only uncorrelated normal random variables have been generated. For nonnormal and correlated variables, the limit state surface, which is determined point by point as explained in the previous section, is transformed to the standardized space of uncorrelated normal variables using the equivalent normal transformation (i.e., the Rackwitz-Fiessler equations) for each couple of $(c, \varphi)$. The two equations used for the transformation of each $(c, \varphi)$ of the limit state surface from the physical space to the standarized normal uncorrelated space $\left(u_{1}, u_{2}\right)$ are (Lemaire 2005)

$$
\begin{gathered}
u_{1}=\left(\frac{c-\mu_{c}^{N}}{\sigma_{c}^{N}}\right) \\
u_{2}=\frac{1}{\sqrt{1-\rho^{2}}}\left[\left(\frac{\varphi-\mu_{\varphi}^{N}}{\sigma_{\varphi}^{N}}\right)-\rho\left(\frac{c-\mu_{c}^{N}}{\sigma_{c}^{N}}\right)\right]
\end{gathered}
$$

where $\rho=$ coefficient of correlation of $c$ and $\varphi$; and $\mu_{c}^{N}, \mu_{\varphi}^{N}, \sigma_{c}^{N}$, and $\sigma_{\varphi}^{N}=$ respectively, the equivalent normal means and standard deviations of the random variables $c$ and $\varphi$. They are determined from the translation approach using the following equations:

$$
\begin{gathered}
\frac{c-\mu_{c}^{N}}{\sigma_{c}^{N}}=\Phi^{-1}\left[F_{c}(c)\right] \\
\frac{\varphi-\mu_{\varphi}^{N}}{\sigma_{\varphi}^{N}}=\Phi^{-1}\left[F_{\varphi}(\varphi)\right]
\end{gathered}
$$

where $F_{c}$ and $F_{\varphi}=$ non-Gaussian cumulative distribution functions of $c$ and $\varphi$; and $\Phi^{-1}(\cdot)=$ inverse of the standard normal cumulative distribution. If desired, the original correlation matrix $\left(\rho_{i j}\right)$ of the nonnormals can be modified to $\rho_{i j}^{\prime}$ in line with the equivalent 
Table 1. Reliability Index and Sensitivity Factors

\begin{tabular}{|c|c|c|c|c|c|c|c|}
\hline$P_{S}(\mathrm{kN} / \mathrm{m})$ & $c^{*}(\mathrm{kPa})$ & $\varphi^{*}(\mathrm{deg})$ & $\beta_{\mathrm{HL}}$ & $\gamma\left(\mu_{c}\right)$ & $\gamma\left(\mu_{\varphi}\right)$ & $F_{c}$ & $F_{\varphi}$ \\
\hline \multicolumn{8}{|c|}{ (a) Uncorrelated normal variables } \\
\hline 500 & 8.87 & 20.69 & 4.17 & 1.30 & 1.39 & 2.25 & 1.53 \\
\hline 700 & 12.45 & 22.00 & 3.27 & 1.21 & 1.60 & 1.61 & 1.43 \\
\hline 900 & 14.76 & 23.47 & 2.54 & 1.16 & 1.81 & 1.35 & 1.33 \\
\hline 1,100 & 16.35 & 24.84 & 1.95 & 1.13 & 2.01 & 1.22 & 1.25 \\
\hline 1,300 & 17.49 & 26.08 & 1.45 & 1.11 & 2.20 & 1.14 & 1.18 \\
\hline 1,500 & 18.35 & 27.19 & 1.02 & 1.09 & 2.38 & 1.09 & 1.12 \\
\hline 1,700 & 19.00 & 28.17 & 0.66 & 1.08 & 2.56 & 1.05 & 1.08 \\
\hline 1,900 & 19.52 & 29.06 & 0.34 & 1.08 & 2.74 & 1.02 & 1.04 \\
\hline 2,100 & 19.93 & 29.86 & 0.05 & 1.02 & 2.91 & 1.00 & 1.01 \\
\hline $2,136.72$ & 20.00 & 30.00 & 0.00 & - & - & 1.00 & 1.00 \\
\hline \multicolumn{8}{|c|}{ (b) Correlated normal variables } \\
\hline 500 & 10.80 & 19.41 & 5.87 & 0.92 & 0.99 & 1.85 & 1.64 \\
\hline 700 & 16.55 & 19.88 & 4.48 & 0.88 & 1.17 & 1.21 & 1.60 \\
\hline 900 & 18.89 & 21.66 & 3.38 & 0.87 & 1.36 & 1.06 & 1.45 \\
\hline 1,100 & 19.89 & 23.46 & 2.53 & 0.87 & 1.54 & 1.01 & 1.33 \\
\hline 1,300 & 20.29 & 25.09 & 1.85 & 0.87 & 1.72 & 0.99 & 1.23 \\
\hline 1,500 & 20.40 & 26.51 & 1.29 & 0.87 & 1.90 & 0.98 & 1.16 \\
\hline 1,700 & 20.36 & 27.76 & 0.82 & 0.87 & 2.07 & 0.98 & 1.10 \\
\hline 1,900 & 20.22 & 28.86 & 0.41 & 0.88 & 2.23 & 0.99 & 1.05 \\
\hline 2,100 & 20.04 & 29.83 & 0.06 & 0.93 & 2.40 & 1.00 & 1.01 \\
\hline $2,136.72$ & 20.00 & 30.00 & 0.00 & - & - & 1.00 & 1.00 \\
\hline \multicolumn{8}{|c|}{ (c) Uncorrelated nonnormal variables } \\
\hline 500 & 12.46 & 18.35 & 4.59 & 1.20 & 5.44 & 1.61 & 1.74 \\
\hline 700 & 14.39 & 20.98 & 3.41 & 1.18 & 5.27 & 1.39 & 1.51 \\
\hline 900 & 15.76 & 23.02 & 2.58 & 1.15 & 4.87 & 1.27 & 1.36 \\
\hline 1,100 & 16.80 & 24.67 & 1.94 & 1.13 & 3.89 & 1.19 & 1.26 \\
\hline 1,300 & 17.60 & 26.04 & 1.42 & 1.12 & 3.51 & 1.14 & 1.18 \\
\hline 1,500 & 18.25 & 27.22 & 0.99 & 1.12 & 3.29 & 1.10 & 1.12 \\
\hline 1,700 & 18.77 & 28.25 & 0.62 & 1.13 & 3.16 & 1.07 & 1.07 \\
\hline 1,900 & 19.21 & 29.15 & 0.30 & 1.19 & 3.03 & 1.04 & 1.04 \\
\hline 2,100 & 19.59 & 29.96 & 0.02 & 1.37 & 2.17 & 1.02 & 1.00 \\
\hline $2,114.15$ & 19.61 & 30.00 & 0.00 & - & - & 1.02 & 1.00 \\
\hline \multicolumn{8}{|c|}{ (d) Correlated nonnormal variables } \\
\hline 500 & 14.99 & 16.77 & 6.20 & 0.89 & 4.03 & 1.33 & 1.92 \\
\hline 700 & 17.41 & 19.45 & 4.51 & 0.89 & 3.99 & 1.15 & 1.63 \\
\hline 900 & 18.82 & 21.68 & 3.34 & 0.89 & 3.76 & 1.06 & 1.45 \\
\hline 1,100 & 19.57 & 23.59 & 2.47 & 0.89 & 3.05 & 1.02 & 1.32 \\
\hline 1,300 & 19.92 & 25.22 & 1.79 & 0.89 & 2.80 & 1.00 & 1.23 \\
\hline 1,500 & 20.01 & 26.64 & 1.23 & 0.90 & 2.66 & 1.00 & 1.15 \\
\hline 1,700 & 19.96 & 27.88 & 0.77 & 0.92 & 2.57 & 1.00 & 1.09 \\
\hline 1,900 & 19.82 & 28.97 & 0.37 & 0.97 & 2.49 & 1.01 & 1.04 \\
\hline 2,100 & 19.63 & 29.95 & 0.02 & 1.13 & 1.80 & 1.02 & 1.00 \\
\hline $2,114.15$ & 19.61 & 30.00 & 0.00 & - & - & 1.02 & 1.00 \\
\hline
\end{tabular}

normal transformation, as suggested in Der Kiureghian and Liu (1986). Some tables of the ratio $\rho_{i j}^{\prime} / \rho_{i j}$ are given in Appendix B2 of Melchers (1999), including closed form solution for the special case of lognormals. For the cases illustrated herein, the correlation matrix, thus modified, differs only slightly from the original correlation matrix. Hence, for simplicity, the examples in this study retain their original unmodified correlation matrices.

The importance sampling density function used is given in the standard uncorrelated space as follows (Lemaire 2005):

$$
f(u)=\frac{1}{\sqrt{2 \pi}} e^{-1 / 2\left(u-u^{*}\right)^{2}}
$$

where $u^{*}=$ transformed value of the design point in the standard uncorrelated space of the random variables. The convergence of the failure probability calculated by IS is achieved for a sample size of 20,000. The corresponding coefficient of variation is about $2 \%$, which is smaller than the commonly adopted value used in 


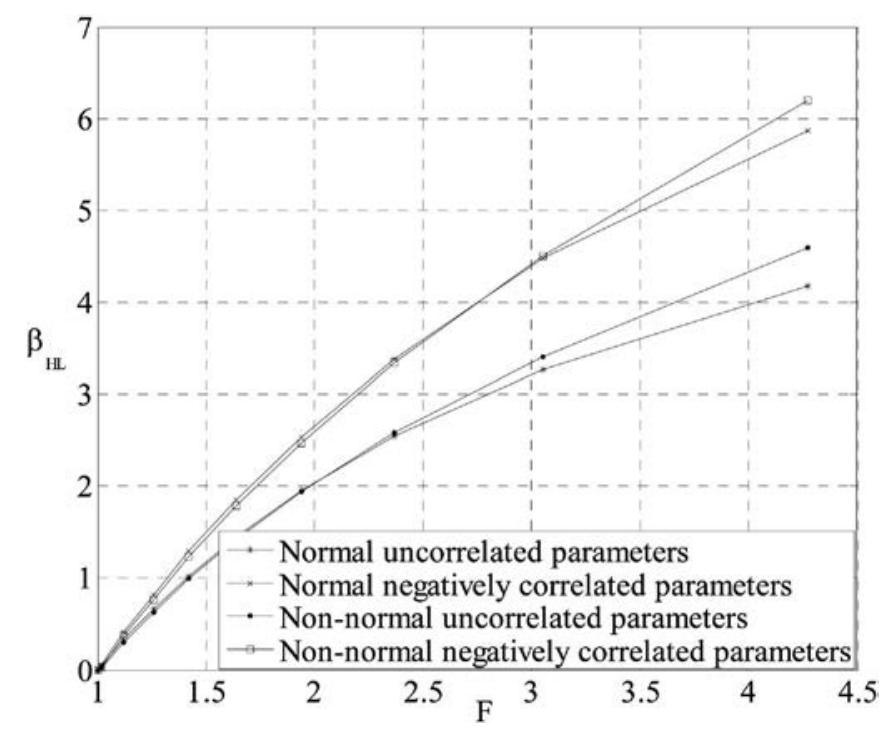

Fig. 4. Reliability index versus safety factor for $\mathrm{COV}_{c}=20 \%$, $\mathrm{COV}_{\varphi}=10 \%$

the literature (i.e., 10\%). For the Monte Carlo simulation, a sample size of 5,000,000 is needed to achieve a constant value of the failure probability and a corresponding coefficient of variation of $2 \%$. In order to have a clear visualization of the convergence of the IS method, the maximal number of samples represented on the $x$-axis of Figs. 6 and 7 was limited to 200,000. In the following, only IS simulation method will be used since it gives accurate results with reasonable sample size. All the subsequent calculations are performed for a coefficient of variation of the estimator of $2 \%$.

By varying the target of the ultimate load, the reliability index is calculated and the CDF is plotted in Figs. 8 and 9 using FORM approximation and importance sampling (IS) simulation. From these figures, it is observed that the CDFs obtained from FORM approximation are in good agreement with those obtained from IS simulation for normal, nonnormal, uncorrelated, and correlated variables for the commonly used values of the coefficients of variation of the soil shear strength parameters (i.e., $\mathrm{COV}_{c}=20 \%$, $\left.\mathrm{COV}_{\varphi}=10 \%\right)$. This means that FORM approximation is an acceptable approach for estimating the failure probability for the commonly used values of the soil variability. In order to explain

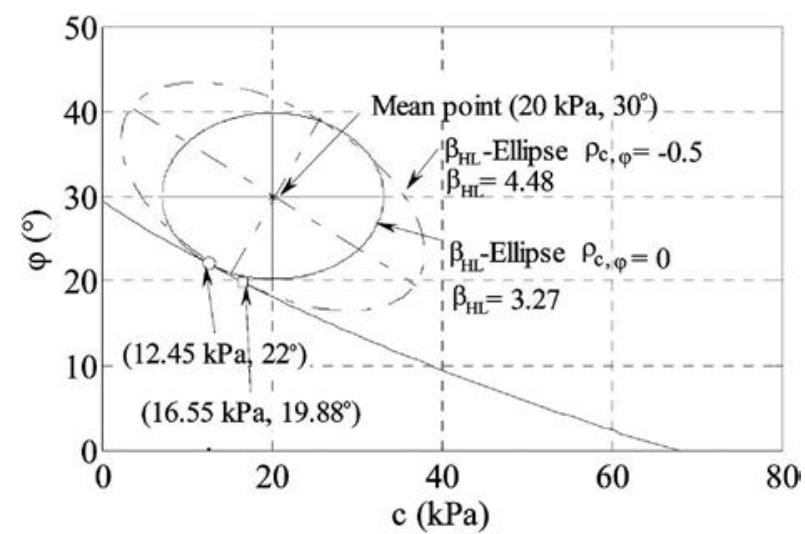

Fig. 5. $\beta_{\mathrm{HL}}$-ellipses for correlated and uncorrelated variables in the physical space

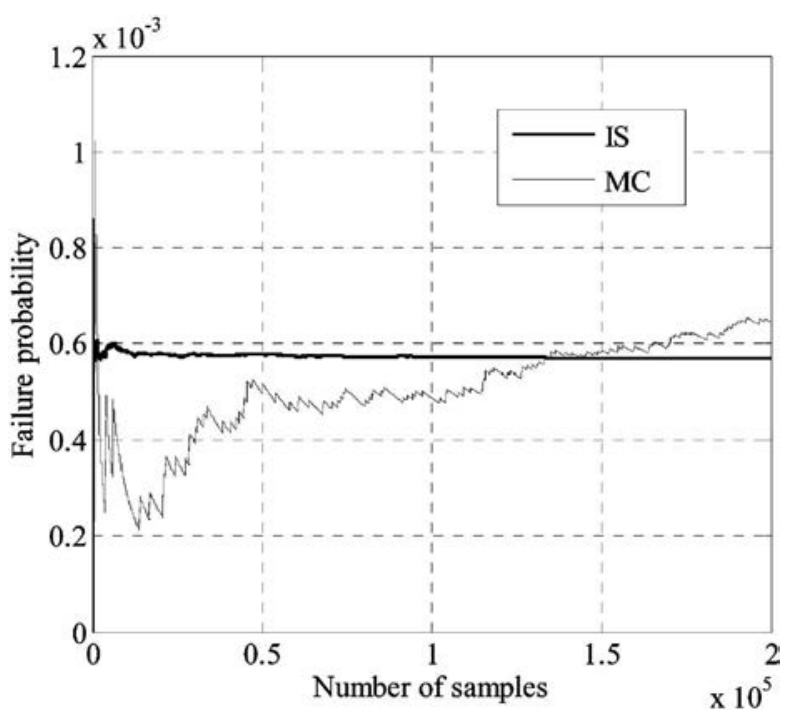

Fig. 6. Failure probability versus the number of samples

the good agreement between the two approaches, the limit state surface is plotted. Fig. 10 shows the limit state surface corresponding to an applied load $P_{S}=1,300 \mathrm{kN} / \mathrm{m}$ for nonnormal correlated random variables in the standard space of normal uncorrelated variables. This figure also shows the linear FORM approximation, which is tangent to the limit state surface at the design point. From this figure, it can be shown that the linear FORM approximation is very close to the exact limit state surface within the circle centred at the origin of the rotated and transformed space and having a radius equal to 3 . This explains why a good agreement between the two approaches is obtained. The difference between the two failure probabilities shown in Fig. 10 is $1.25 \%$. For larger values of the coefficients of variation (i.e., $\mathrm{COV}_{c}=50 \%, \mathrm{COV}_{\varphi}=20 \%$ ), the difference between the two approaches increases and attains $3.32 \%$ as can be shown from the values given in Fig. 11. This can be explained by the curvature of the limit state surface near the design point. Since the limit state surface is convex with respect to the origin of the rotated and transformed space, the FORM approximation overestimates the failure probability.

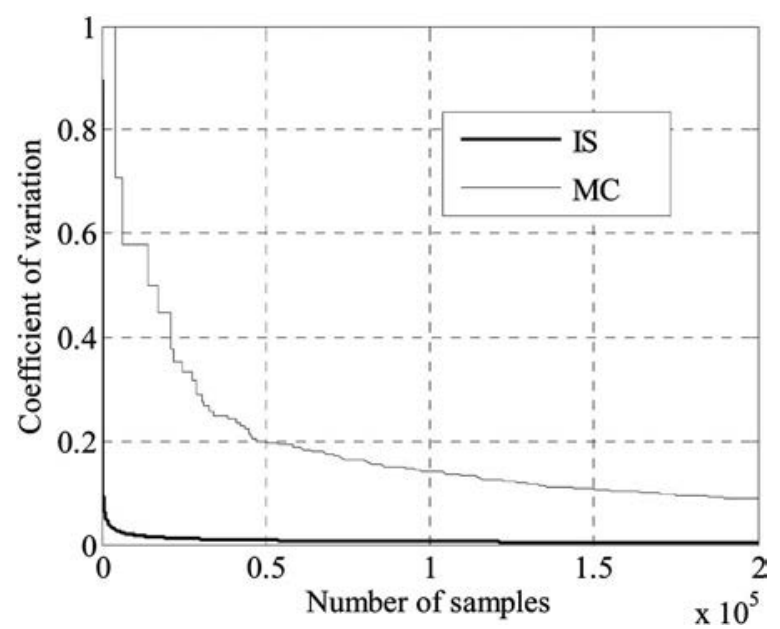

Fig. 7. Coefficient of variation of the failure probability versus the number of samples 


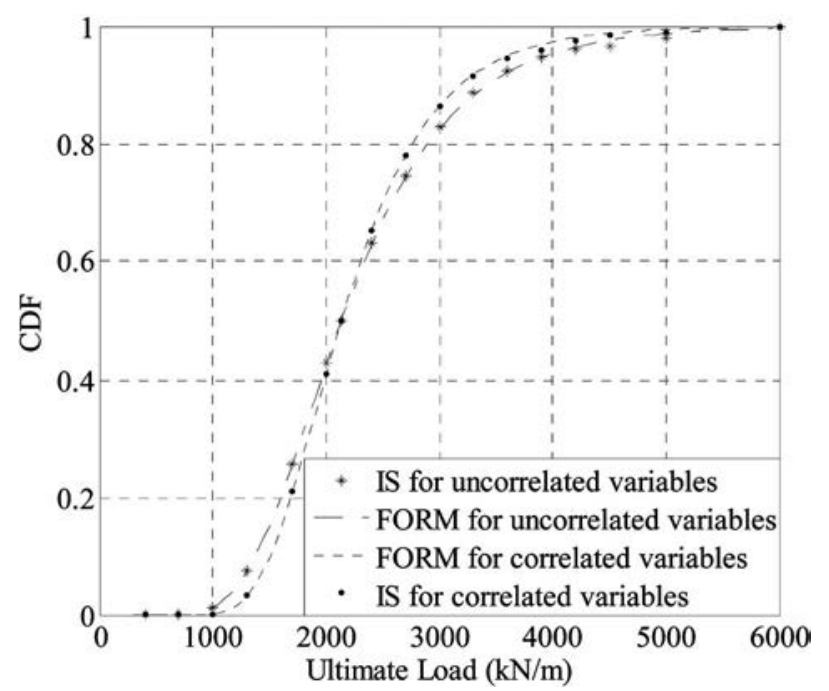

Fig. 8. Comparison of the CDFs of the ultimate load given by FORM and IS for normal variables

Fig. 12 presents the CDFs of the ultimate load for normal, nonnormal, correlated, and uncorrelated variables as given by FORM. When no correlation between shear strength parameters is considered, one can notice a more spread out CDF of the ultimate load (i.e., a higher coefficient of variation of the ultimate load). The chosen probability distribution (i.e., normal, lognormal, and beta distribution) does not significantly affect the values of the failure probability.

Fig. 13 presents the effect of the coefficient of variation of the shear strength parameters on the CDF of the ultimate load. It can be seen that a small change in the coefficient of variation of $\varphi$ highly affects the CDF curve. On the other hand, the CDF curve is not sensitive to changes in the uncertainty of the cohesion. Thus, the failure probability is highly influenced by the coefficient of variation of $\varphi$. The greater the scatter in $\varphi$, the higher the failure probability. This means that accurate determination of the uncertainties of the angle of internal friction $\varphi$ is very important

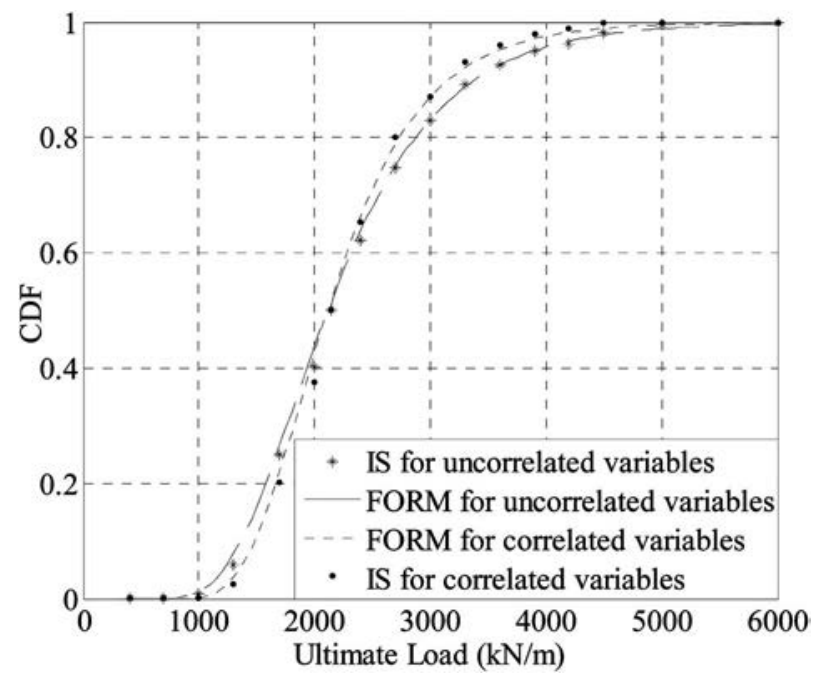

Fig. 9. Comparison of the CDFs of the ultimate load given by FORM and IS for nonnormal variables

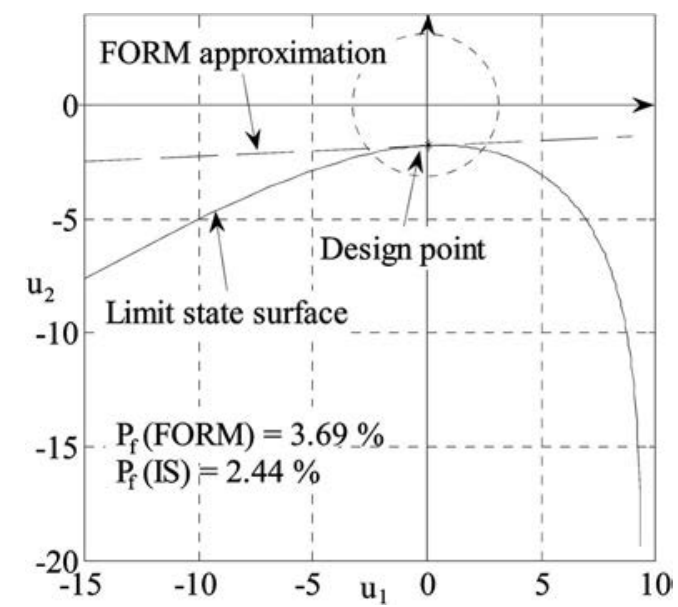

Fig. 10. Limit state surface and FORM approximation for nonnormal correlated variables, for $\mathrm{COV}_{c}=20 \%, \mathrm{COV}_{\varphi}=10 \%$

in obtaining reliable probabilistic results. In contrast, the coefficient of variation of $c$ does not significantly affect the failure probability.

\section{Reliability-Based Design}

The conventional approach used in the design of a shallow foundation is to prescribe a target safety factor (generally $F=3$ ) and to determine the corresponding breadth $B$ of the footing. Recently, a reliability-based design approach (RBD) has been used by several authors [e.g., Low (2005) and Phoon et al. (2003), among others]. This approach was used in this section. It consists of the calculation of $B$ for a target reliability index of 3.8 as suggested by Eurocode 7 for the ultimate limit states (Calgaro 1996). This foundation breadth is called hereafter "probabilistic foundation breadth."

Fig. 14 presents the probabilistic foundation breadth for different values of the coefficients of variation of the shear strength parameters and their coefficient of correlation. This figure also presents the deterministic breadth corresponding to a safety factor

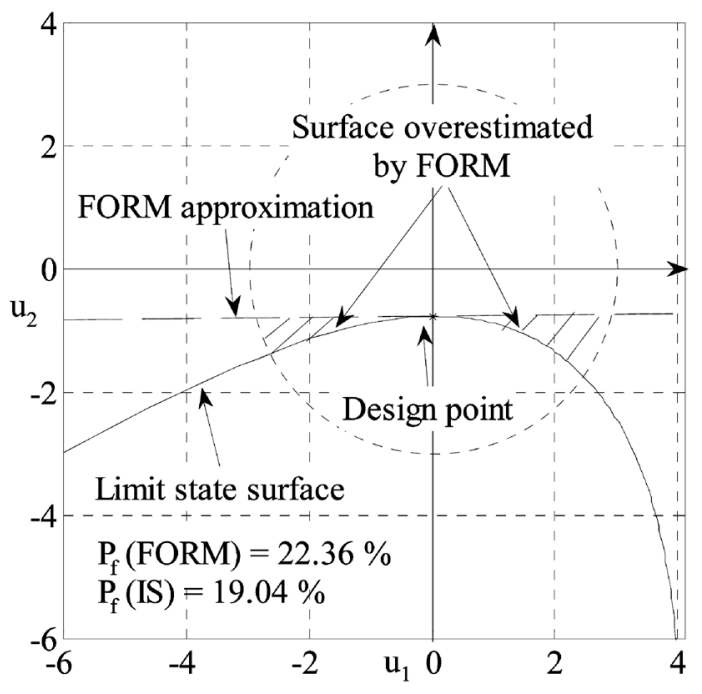

Fig. 11. Limit state surface and FORM approximation for nonnormal correlated variables and large coefficients of variation $\mathrm{COV}_{c}=50 \%$, $\mathrm{COV}_{\varphi}=20 \%$ 


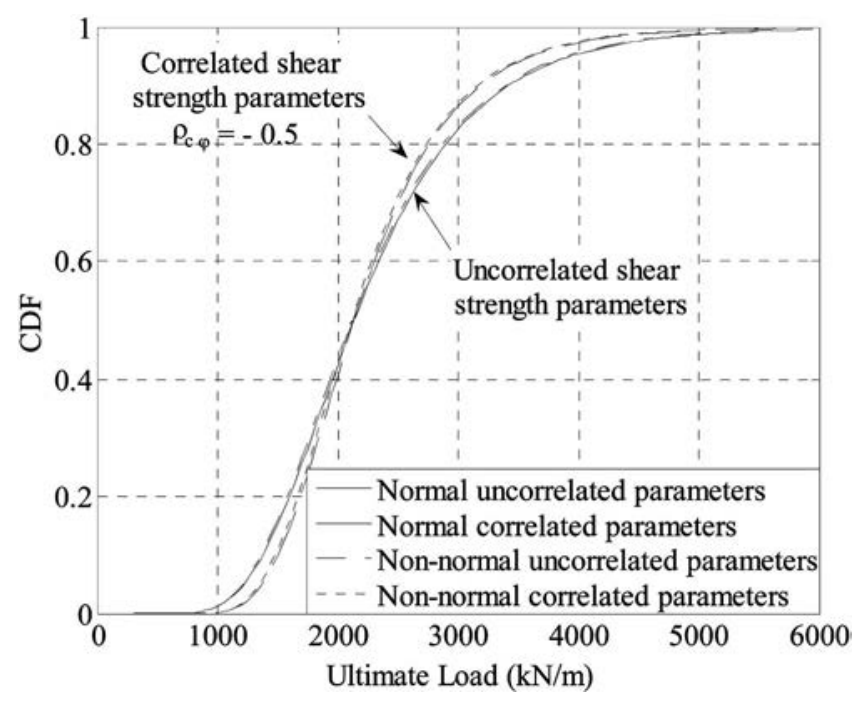

Fig. 12. Comparison of the CDFs of the ultimate load for normal, nonnormal, correlated, and uncorrelated variables

of 3. The probabilistic foundation breadth decreases with the increase of the negative correlation between the shear strength parameters and the decrease of their coefficients of variation. It can become smaller than the deterministic breadth for the common values of the soil variability (i.e., $\operatorname{COV}_{\varphi}=10 \%, \operatorname{COV}_{c}=20 \%$, $\left.-0.7 \leqslant \rho_{c, \varphi} \leqslant-0.3\right)$. For high values of the coefficients of variation and small correlation coefficient, the design breadth for normal variables is higher than that of nonnormal variables. This means that, for the case in hand, assuming normal distributions for the random variables is more conservative than assuming nonnormal distributions. As a conclusion, the deterministic footing breadth may be higher or lower than the reliability-based footing width, depending on the uncertainties.

Contrary to Eurocode 7, which prescribes constant values of the partial safety factors $F_{c}$ and $F_{\varphi}$, the present RBD has the advantage of providing different values of these factors depending on the soil variability. These factors are the optimal ones and are determined rigorously by a maximization of the failure probability for a given soil variability. Hence, a reliability-based design has the merit of explicitly reflecting the correlation structure, the

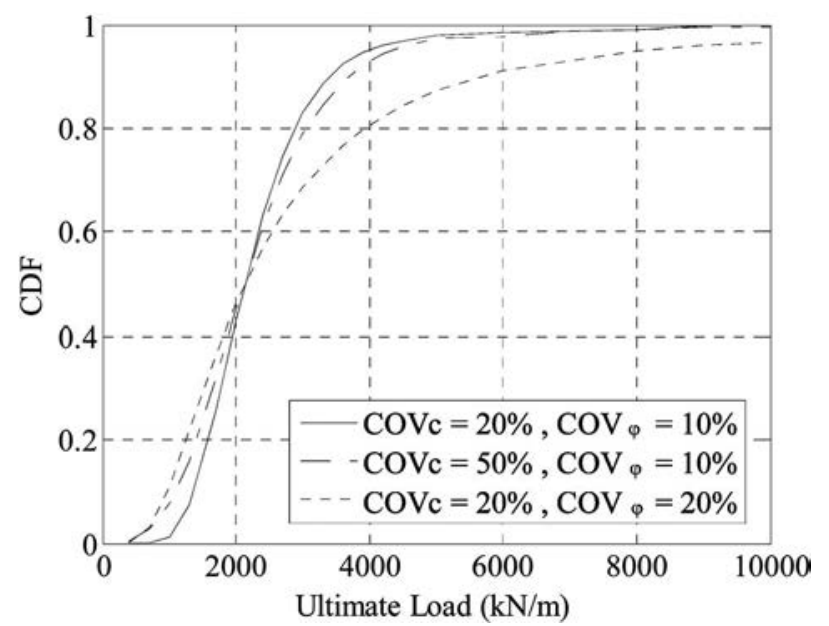

Fig. 13. Comparison of CDFs of the ultimate load for different values of the coefficients of variation

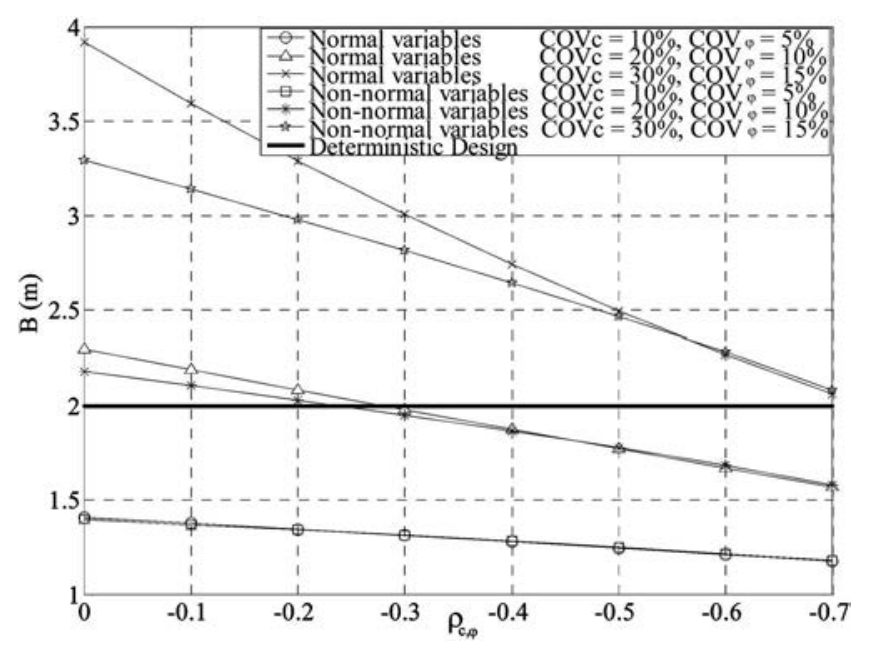

Fig. 14. Comparison between probabilistic and deterministic design

standard deviations, and the probability distributions of the underlying random variables, and of automatically seeking the design point without relying on prescribed values of partial safety factors.

\section{Mechanism M2}

The number of rigid blocks used in the $M 2$ mechanism (Fig. 3) is equal to 12 since further increase in the number of blocks improves the optimal solution by less than $0.5 \%$. For the seismic coefficient, an exponential distribution $(\operatorname{Exp} D)$ and an extreme value type II distribution (EVD) are used (Haldar and Mahadevan 2000). The mean value of the horizontal seismic coefficient is assumed to be 0.15 . The angle of internal friction is considered to follow the beta distribution.

It was shown for the $M 1$ mechanism (Fig. 13) that the failure probability of a vertically loaded footing is more sensitive to a variation of the angle of internal friction than the cohesion. For the seismically loaded footing in hand, Fig. 15 presents the effect of the randomness of the soil cohesion on the reliability index for both exponential and extreme value distributions of the seismic coefficient. In this figure, the cohesion $c$ is assumed to be either a lognormally distributed random variable with the commonly used

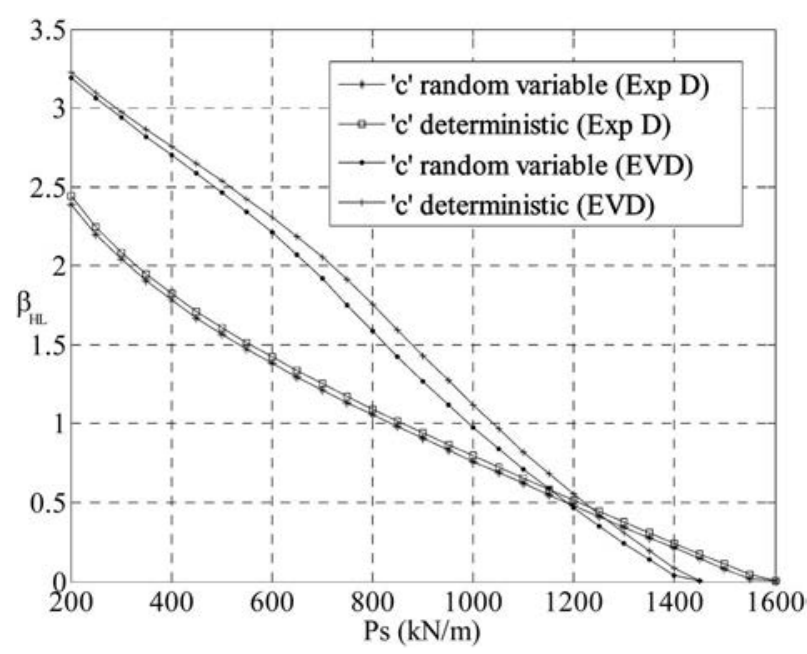

Fig. 15. Effect of the variability of $c$ on the reliability index 
Table 2. Probabilistic Results When $K_{h}$ Follows the Exponential Distribution

\begin{tabular}{|c|c|c|c|c|c|c|}
\hline $\begin{array}{l}P_{S} \\
\mathrm{kN} / \mathrm{m} \\
\end{array}$ & $\begin{array}{c}\varphi^{*} \\
(\mathrm{deg}) \\
\end{array}$ & $K_{h}^{*}$ & $\beta_{\mathrm{HL}}$ & $\gamma\left(\mu_{\varphi}\right)$ & $\gamma\left(\mu_{K_{h}}\right)$ & $P_{f}(\%)$ \\
\hline 200 & 28.58 & 0.70 & 2.44 & 1.02 & 2.03 & 3.87 \\
\hline 450 & 28.77 & 0.45 & 1.71 & 1.03 & 2.62 & 6.52 \\
\hline 700 & 28.84 & 0.32 & 1.25 & 1.06 & 1.95 & 11.45 \\
\hline 1,000 & 29.01 & 0.22 & 0.80 & 1.09 & 1.34 & 21.41 \\
\hline 1,300 & 29.40 & 0.15 & 0.37 & 1.20 & 0.47 & 35.33 \\
\hline 1,600 & 30.01 & 0.10 & 0.00 & - & - & 50.00 \\
\hline
\end{tabular}

coefficient of variation $(20 \%)$ or as a deterministic parameter with value equal to the mean value (i.e., $20 \mathrm{kPa}$ ). One may note that whether $c$ is random or deterministic has only a minor effect on the value of the reliability index.

Reliability Index, Failure Probability, and Sensitivity Factors Tables 2 and 3 present the reliability results (i.e., Hasofer-Lind reliability index, the corresponding design point, the omission sensitivity factors, and the failure probability) for different values of the applied load $P_{S}$ varying from small values up to the deterministic ultimate load defined earlier for both exponential and extreme value distributions on $K_{h}$. The reliability index decreases with the increase of the vertical applied load $P_{S}$. The exponential distribution on $K_{h}$ gives more conservative results than the extreme value distribution on $K_{h}$. The values of the omission sensitivity factors suggest that, when the applied load is relatively small compared to the deterministic ultimate load, whether $\varphi$ is random or deterministic has only a minor effect on the reliability index since $\gamma\left(\mu_{\varphi}\right) \approx 1$ for both types of the probability distributions on $K_{h}$. On the other hand, the randomness of the seismic coefficient has a significant effect on the reliability index. Hence, for small values of the applied load, one can neglect the uncertainty in $\varphi$. In contrast, for higher values of the applied load, the uncertainties of both the angle of internal friction and the seismic coefficient should be considered in the reliability analysis.

\section{Probability Distribution of the Punching Safety Factor}

Fig. 16 shows the CDF of the punching safety factor obtained from FORM approximation for the exponential distribution and for different values of the coefficient of variation of the extreme value distribution. It can be seen that the choice of the probability distribution on $K_{h}$ significantly affects the probability distribution of the safety factor. The dispersion of $F$ is significantly larger when an exponential distribution is assumed for the seismic coefficient.

Table 3. Probabilistic Results When $K_{h}$ Follows the Extreme Value Distribution and $\mathrm{COV}_{K h}=40 \%$

\begin{tabular}{|c|c|c|c|c|c|c|}
\hline $\begin{array}{l}P_{S} \\
\mathrm{kN} / \mathrm{m} \\
\end{array}$ & $\begin{array}{c}\varphi^{*} \\
(\mathrm{deg})\end{array}$ & $K_{h}^{*}$ & $\beta_{\mathrm{HL}}$ & $\gamma\left(\mu_{\varphi}\right)$ & $\gamma\left(\mu_{K_{h}}\right)$ & $P_{f}(\%)$ \\
\hline 200 & 28.86 & 0.71 & 3.22 & 1.02 & 1.54 & 0.06 \\
\hline 450 & 28.08 & 0.44 & 2.65 & 1.02 & 1.69 & 0.41 \\
\hline 700 & 26.90 & 0.27 & 2.05 & 1.10 & 1.18 & 2.00 \\
\hline 1,000 & 27.08 & 0.16 & 1.11 & 1.45 & 0.97 & 13.25 \\
\hline 1,300 & 29.15 & 0.14 & 0.31 & 2.18 & 0.54 & 37.96 \\
\hline 1,450 & 30.00 & 0.13 & 0.00 & - & - & 50.00 \\
\hline
\end{tabular}

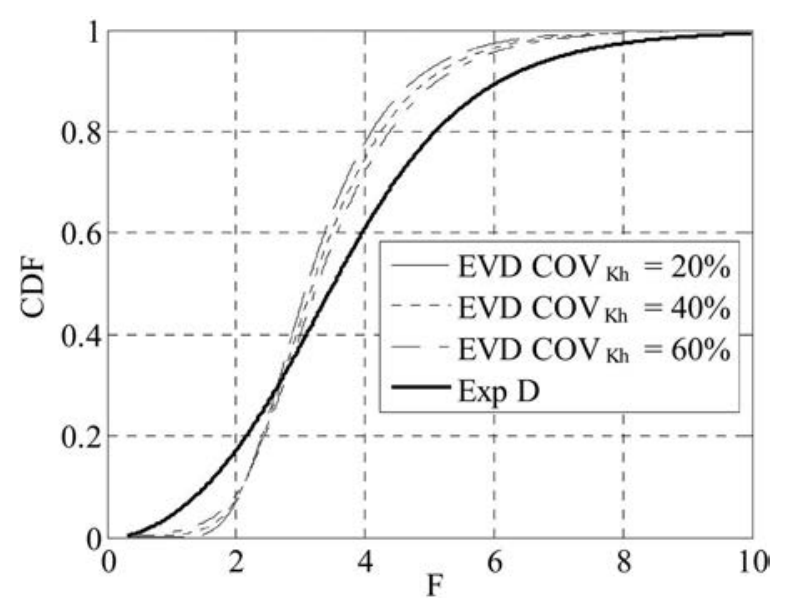

Fig. 16. CDF of the punching safety factor for $P_{S}=450 \mathrm{kN} / \mathrm{m}$

\section{Probabilistic Design}

For the design of a vertically loaded footing situated in seismic area, a reasonable target failure probability of $1 \%$ is used. Fig. 17 presents the probabilistic foundation breadth for the extreme value distribution on $K_{h}$ and for different values of the coefficient of variation of $K_{h}$. The deterministic breadth corresponding to a safety factor of 3 is also shown. The applied load is $700 \mathrm{kN} / \mathrm{m}$. The probabilistic foundation breadth increases with the increase of the coefficient of variation of $K_{h}$. It can be greater than the deterministic breadth for large values of the coefficient of variation.

\section{Conclusion}

A reliability-based analysis and design of a shallow strip foundation subjected to a vertical and a pseudostatic horizontal seismic loading was performed. Only the punching failure mode of the ultimate limit state was studied. Two rigorous deterministic models based on limit analysis failure mechanisms were used.

For the vertically loaded footing, the reliability index calculated based on the critical probabilistic surface is more critical than that determined using the critical deterministic surface. The reliability index decreases with the increase of the applied load $P_{S}$. The assumption of uncorrelated shear strength parameters is

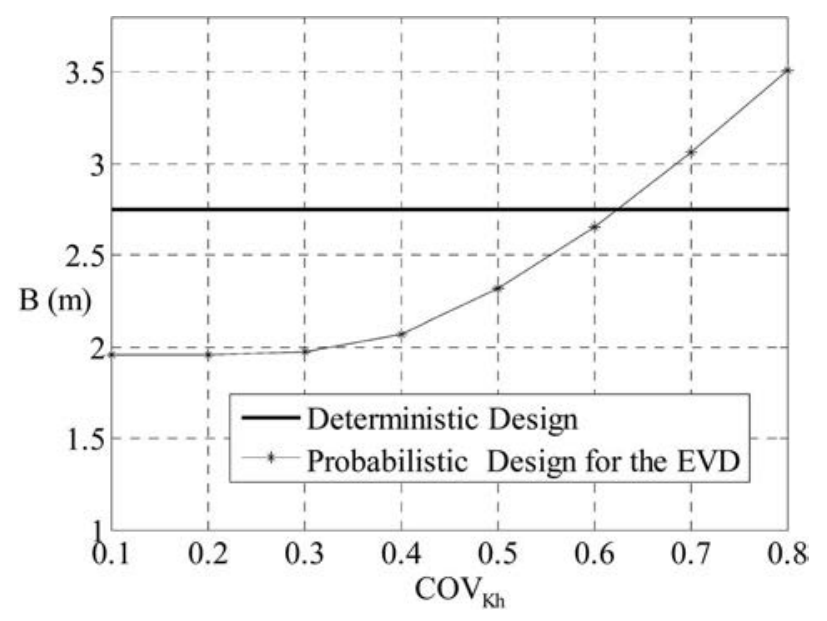

Fig. 17. Comparison between deterministic and probabilistic design 
conservative in comparison to that of negatively correlated parameters. The reliability index is much more sensitive to $\varphi$ than to $c$, especially for the case of nonnormal variables. The greater the scatter in $\varphi$, the higher the failure probability. This means that the accurate determination of the uncertainties of the angle of internal friction $\varphi$ is important in obtaining reliable probabilistic results. FORM approximation is an acceptable approach for estimating the failure probability for the commonly used values of the soil variability. When no correlation between shear strength parameters is considered, a more spread out CDF of the ultimate load was obtained. For the case in hand, the chosen probability distribution does not significantly affect the values of the failure probability. The probabilistically-designed foundation breadth decreases with the increase of the negative correlation between the shear strength parameters and the decrease of their coefficients of variation. Contrary to Eurocode 7, which prescribes constant values of the partial safety factors $F_{c}$ and $F_{\varphi}$, a reliability-based design has the advantage of providing different values of these factors depending on the soil variability and correlation structure. These factors are by-products of a reliabilitybased design and reflect parametric sensitivities from case to case in a way rigid partial safety factors cannot.

For the seismically loaded footing, the extreme value distribution was used to model the uncertainties of $K_{h}$. For values of the vertical load much below the deterministic ultimate load, one can neglect the uncertainty in $\varphi$. In contrast, for higher values of the vertical load, the uncertainties of both $\varphi$ and $K_{h}$ should be considered in the reliability analysis.

\section{Acknowledgments}

The first two writers would like to thank the Lebanese National Council for Scientific Research (CNRSL) and the French organization EGIDE for providing the financial support for this research.

\section{Appendix I}

The expressions of the functions $f_{i}(i=1, \ldots, 6)$ are given as follows:

$$
\begin{gathered}
f_{1}=\frac{\tan \theta}{2} \\
f_{2}=\frac{\cos (\theta-\varphi)}{2 \cos ^{2} \theta \sin \left(\beta_{1}-2 \varphi\right)} \\
\cdot \sum_{i=1}^{n}\left[\frac{\sin \alpha_{i} \sin \beta_{i}}{\sin \left(\alpha_{i}+\beta_{i}\right)} \sin \left(\beta_{i}-\theta-\sum_{j=1} \alpha_{j}-\varphi\right)\right. \\
\left.\cdot \prod_{j=1}^{i-1} \frac{\sin ^{2} \beta_{j} \sin \left(\alpha_{j}+\beta_{j}-2 \varphi\right)}{\sin ^{2}\left(\alpha_{j}+\beta_{j}\right) \sin \left(\beta_{j+1}-2 \varphi\right)}\right] \\
f_{3}=\frac{\cos (\theta-\varphi)}{\cos \theta \sin \left(\beta_{1}-2 \varphi\right)} \frac{\sin \beta_{n}}{\sin \left(\alpha_{n}+\beta_{n}\right)} \\
\cdot \sin \left(\beta_{n}-\theta-\sum_{j=1} \alpha_{j}-\varphi\right) \\
\quad \prod_{j=1}^{n-1} \frac{\sin \beta_{j} \sin \left(\alpha_{j}+\beta_{j}-2 \varphi\right)}{\sin \left(\alpha_{j}+\beta_{j}\right) \sin \left(\beta_{j+1}-2 \varphi\right)}
\end{gathered}
$$

$$
f_{4}=\frac{\cos (\varphi) \cos \left(\beta_{1}-\theta-\varphi\right)}{2 \cos \theta \sin \left(\beta_{1}-2 \varphi\right)}
$$

$$
\begin{aligned}
& f_{5}= \frac{\cos (\theta-\varphi) \cos (\varphi)}{2 \cos \theta \sin \left(\beta_{1}-2 \varphi\right)} \\
& \cdot \sum_{i=1}^{n}\left[\frac{\sin \alpha_{i}}{\sin \left(\alpha_{i}+\beta_{i}\right)} \prod_{j=1}^{i-1} \frac{\sin \beta_{j} \sin \left(\alpha_{j}+\beta_{j}-2 \varphi\right)}{\sin \left(\alpha_{j}+\beta_{j}\right) \sin \left(\beta_{j+1}-2 \varphi\right)}\right] \\
& f_{6}=\frac{\cos (\theta-\varphi) \cos (\varphi)}{2 \cos \theta \sin \left(\beta_{1}-2 \varphi\right)} \\
& \cdot \sum_{i=2}^{n}\left[\frac{\sin \left(\beta_{i-1}-\beta_{i}+\alpha_{i-1}\right)}{\sin \left(\beta_{i}-2 \varphi\right)} \cdot \prod_{j=1}^{i-1} \frac{\sin \beta_{j}}{\sin \left(\alpha_{j}+\beta_{j}\right)}\right. \\
&\left.\quad \cdot \prod_{j=1}^{i-2} \frac{\sin \left(\alpha_{j}+\beta_{j}-2 \varphi\right)}{\sin \left(\beta_{j+1}-2 \varphi\right)}\right]
\end{aligned}
$$

\section{Appendix II}

The expressions of the functions $g_{i}(i=1, \ldots, 6)$ are given as follows:

$$
\begin{aligned}
& g_{1}=\frac{\sin ^{2} \beta_{1}}{\sin ^{2}\left(\alpha_{1}+\beta_{1}\right)} \sum_{i=1}^{n}\left[\frac{\sin \alpha_{i} \sin \left(\alpha_{i}+\beta_{i}\right)}{\sin \beta_{i}} \sin \left(\beta_{i}-\varphi-\sum_{j=1}^{i-1} \alpha_{j}\right)\right. \\
& \left.\times \prod_{j=2}^{i} \frac{\sin ^{2} \beta_{j}}{\sin ^{2}\left(\alpha_{j}+\beta_{j}\right)} \prod_{j=1}^{i-1} \frac{\sin \left(\alpha_{j}+\beta_{j}-2 \varphi\right)}{\sin \left(\beta_{j+1}-2 \varphi\right)}\right] \\
& g_{2}=\frac{\sin ^{2} \beta_{1}}{\sin ^{2}\left(\alpha_{1}+\beta_{1}\right)} \sum_{i=1}^{n}\left[\frac{\sin \alpha_{i} \sin \left(\alpha_{i}+\beta_{i}\right)}{\sin \beta_{i}} \cos \left(\beta_{i}-\varphi-\sum_{j=1}^{i-1} \alpha_{j}\right)\right. \\
& \left.\times \prod_{j=2}^{i} \frac{\sin ^{2} \beta_{j}}{\sin ^{2}\left(\alpha_{j}+\beta_{j}\right)} \prod_{j=1}^{i-1} \frac{\sin \left(\alpha_{j}+\beta_{j}-2 \varphi\right)}{\sin \left(\beta_{j+1}-2 \varphi\right)}\right] \\
& g_{3}=\frac{\sin \beta_{1}}{\sin \left(\alpha_{1}+\beta_{1}\right)} \sin \left(\beta_{n}-\varphi-\sum_{j=1}^{n-1} \alpha_{j}\right) \\
& \times \prod_{j=2}^{n} \frac{\sin \beta_{j}}{\sin \left(\alpha_{j}+\beta_{j}\right)} \prod_{j=1}^{n-1} \frac{\sin \left(\alpha_{j}+\beta_{j}-2 \varphi\right)}{\sin \left(\beta_{j+1}-2 \varphi\right)} \\
& g_{4}=\frac{\sin \beta_{1}}{\sin \left(\alpha_{1}+\beta_{1}\right)} \cos \left(\beta_{n}-\varphi-\sum_{j=1}^{n-1} \alpha_{j}\right) \\
& \times \prod_{j=2}^{n} \frac{\sin \beta_{j}}{\sin \left(\alpha_{j}+\beta_{j}\right)} \prod_{j=1}^{n-1} \frac{\sin \left(\alpha_{j}+\beta_{j}-2 \varphi\right)}{\sin \left(\beta_{j+1}-2 \varphi\right)} \\
& g_{5}=\frac{\sin \beta_{1} \cos \varphi}{\sin \left(\alpha_{1}+\beta_{1}\right)} \sum_{i=1}^{n}\left[\frac{\sin \alpha_{i}}{\sin \beta_{i}} \prod_{j=2}^{i} \frac{\sin \beta_{j}}{\sin \left(\alpha_{j}+\beta_{j}\right)} \prod_{j=1}^{i-1} \frac{\sin \left(\alpha_{j}+\beta_{j}-2 \varphi\right)}{\sin \left(\beta_{j+1}-2 \varphi\right)}\right] \\
& g_{6}=\frac{\sin \beta_{1} \cos \varphi}{\sin \left(\alpha_{1}+\beta_{1}\right)} \sum_{i=1}^{n-1}\left[\frac{\sin \left(\beta_{i}-\beta_{i+1}+\alpha_{i}\right)}{\sin \left(\beta_{i+1}-2 \varphi\right)}\right. \\
& \left.\times \prod_{j=2}^{i} \frac{\sin \beta_{j}}{\sin \left(\alpha_{j}+\beta_{j}\right)} \prod_{j=1}^{i-1} \frac{\sin \left(\alpha_{j}+\beta_{j}-2 \varphi\right)}{\sin \left(\beta_{j+1}-2 \varphi\right)}\right]
\end{aligned}
$$




\section{References}

Calgaro, J. A. (1996). Introduction aux Eurocodes. Sécurité des constructions et bases de la théorie de la fiabilité, Presses de l'ENPC, Paris (in French).

Cherubini, C. (2000). "Reliability evaluation of shallow foundation bearing capacity on $\mathrm{C}^{\prime}, \varphi^{\prime}$ soils." Can. Geotech. J., 37, 264-269.

Christian, J., Ladd, C., and Baecher, G. (1994). "Reliability applied to slope stability analysis." J. Geotech. Engrg., 120(12), 2180-2207.

Der Kiureghian, A., and Liu, P. L. (1986). "Structural reliability under incomplete probability information." J. Eng. Mech., 112(1), 85-104.

Ditlevsen, O. (1981). Uncertainty modelling: With applications to multidimensional civil engineering systems, McGraw-Hill, New York.

El-Ramly, H., Morgenstern, N. R., and Cruden, D. M. (2002). "Probabilistic slope stability analysis for practice." Can. Geotech. J., 39, 665683.

El-Ramly, H., Morgenstern, N. R., and Cruden, D. M. (2003). "Probabilistic stability analysis of a tailing dyke on presheared clay-shale." Can. Geotech. J., 40, 192-208.

Fenton, G. A., and Griffiths, D. V. (2002). "Probabilistic foundation settlement on spatially random soil." J. Geotech. Geoenviron. Eng., 128(5), 381-390.

Fenton, G. A., and Griffiths, D. V. (2003). "Bearing capacity prediction of spatially random C- $\varphi$ soils." Can. Geotech. J., 40, 54-65.

Fenton, G. A., and Griffiths, D. V. (2005). "Three-dimensional probabilistic foundation settlement." J. Geotech. Geoenviron. Eng., 131(2), 232-239.

Griffiths, D. V., and Fenton, G. A. (2001). "Bearing capacity of spatially random soil: The undrained clay Prandtl problem revisited." Geotechnique, 51(4), 351-359.

Griffiths, D. V., Fenton, G. A., and Manoharan, N. (2002). "Bearing capacity of rough rigid strip footing on cohesive soil: Probabilistic study." J. Geotech. Geoenviron. Eng., 128(9), 743-755.

Haldar, A., and Mahadevan, S. (2000). Probability, reliability, and statistical methods in engineering design, Wiley, New York.

Harr, M. E. (1987). Reliability-based design in civil engineering, McGraw-Hill, New York.

Hasofer, A. M., and Lind, N. C. (1974). "Exact and invariant secondmoment code format." J. Engrg. Mech. Div., 100(1), 111-121.

Hassan, A., and Wolff, T. (1999). "Search algorithm for minimum reliability index of earth slopes." J. Geotech. Geoenviron. Eng., 125(4), 301-308.

Lemaire, M. (2005). Fiabilité des structures, Hermes-Lavoisier, Paris (in French).

Low, B. K. (2005). "Reliability-based design applied to retaining walls."
Geotechnique, 55(1), 63-75.

Low, B. K., Gilbert, R. B., and Wright, S. G. (1998). "Slope reliability analysis using generalized method of slices." J. Geotech. Geoenviron. Eng., 124(4), 350-362.

Low, B. K., and Phoon, K. K. (2002). "Practical first-order reliability computations using spreadsheet." Proc., Probabilistics in Geotechnics: Technical and Economic Risk Estimation, 39-46.

Low, B. K., and Tang, W. H. (1997a). "Efficient reliability evaluation using spreadsheet." J. Eng. Mech., 123(7), 749-752.

Low, B. K., and Tang, W. H. (1997b). "Probabilistic slope analysis using Janbu's generalized procedure of slices." Comput. Geotech., 21(2), $121-142$.

Low, B. K., and Tang, W. H. (1997c). "Reliability analysis of reinforced embankments on soft ground." Can. Geotech. J., 34, 672-685.

Low, B. K., and Tang, W. H. (2004). "Reliability analysis using objectoriented constrained optimization." Struct. Safety, 26, 68-89.

Lumb, P. (1970). "Safety factors and the probability distribution of soil strength." Can. Geotech. J., 7, 225-242.

Melchers, R. E. (1999). Structural reliability analysis and prediction, 2nd Ed., Wiley, New York.

Phoon, K.-K. (2004). "General non-Gaussian probability models for firstorder reliability method (FORM)." A state-of-the-art Rep., Int. Centre for Geohazards (ICG), Oslo, Norway.

Phoon, K.-K., and Kulhawy, F. H. (1999). "Evaluation of geotechnical property variability." Can. Geotech. J., 36, 625-639.

Phoon, K.-K., Kulhawy, F. H., and Grigoriu, M. D. (2003). "Multiple resistance factor design for shallow transmission line structure foundations." J. Geotech. Geoenviron. Eng., 129(9), 807-818.

Popescu, R., Deodatis, G., and Nobahar, A. (2005). "Effect of random heterogeneity of soil properties on bearing capacity." Probab. Eng. Mech., 20, 324-341.

Przewlocki, J. (2005). "A stochastic approach to the problem of bearing capacity by the method of characteristics." Comput. Geotech., 32, 370-376.

Rackwitz, R., and Fiessler, B. (1978). "Structural reliability under combined random load sequences." Comput. Struct., 9(5), 484-494.

Soubra, A.-H. (1999). "Upper-bound solutions for bearing capacity of foundations." J. Geotech. Geoenviron. Eng., 125(1), 59-68.

STRUREL. (1991). A structural reliability analysis program: Theoretical manual, RCP GmbH, Munchen, Germany.

Wolff, T. H. (1985). "Analysis and design of embankment dam slopes: A probabilistic approach.” Ph.D. thesis, Purdue Univ., Lafayette, Ind.

Yuceman, M. S., Tang, W. H., and Ang, A. H. S. (1973). "A probabilistic study of safety and design of earth slopes." Civil Engineering Studies, Structural Research Series, 402, Univ. of Illinois, Urbana, Ill. 